\title{
Design and Fabrication of Sidewalls-Extended Electrode Configuration for Ridged Lithium Niobate Electrooptical Modulator
}

\author{
Yi-Kuei Wu, Student Member, IEEE, and Way-Seen Wang, Member, IEEE
}

\begin{abstract}
A ridged lithium niobate electrooptical (EO) modulator with low driving voltage and reduced size is proposed. In particular, the electrodes of the proposed device are extended to the sidewalls of the ridge slopes to make the electric and optical fields much more overlapped than those reported. For a more detailed simulation, the electric and optical fields involved in the overlap integral are numerically calculated rather than by using approximate analytic solutions. Experimental results show that the halfwave voltage is reduced by at least $15 \%$ in comparison to that of an EO modulator without extended electrode configuration. As both the electrode length and gap are decreased, the device sizes can be reduced by about $18 \%$.
\end{abstract}

Index Terms-Electrooptical (EO) modulator, half-wave voltage, lithium niobate, overlap integral, ridge waveguide.

\section{INTRODUCTION}

$\mathbf{E}$ LECTROOPTICAL (EO) modulators are key devices in optical communication system. For an efficient operation of an EO modulator, the reduction of driving voltage is an important issue. In principle, increasing the overlap integral by matching the maximum of electric and optical fields is a good way to reduce the half-wave voltage. However, its effect is limited [1]. Recently, an effective way to reduce the half-wave voltage is realized by using ridge structures and placing electrodes on both sides of the ridge [2], [3]. In this paper, the electrodes are extended to the sidewalls of the ridge slopes to make the electric and optical fields much more overlapped than those reported in [3]. With the proposed structure, experimental results show that the half-wave voltage is reduced by at least $15 \%$ in comparison to that of an EO modulator without extended electrode configuration. As both the electrode length and gap are decreased, the device sizes can be reduced by about $18 \%$.

\section{Simulation}

In principle, the half-wave voltage $V_{\pi}$ of a modulator can be written as [4]

$$
V_{\pi}=\frac{\lambda G}{n^{3} r_{33} L \Gamma}
$$

Manuscript received February 9, 2007; revised August 2, 2007. This work was supported by the National Science Council, Taipei, Taiwan, R.O.C., under Contract NSC 95-2221-E-002-383.

Y.-K. Wu is with the Graduate Institute of Electro-Optical Engineering, National Taiwan University, Taipei 106, Taiwan, R.O.C.

W.-S. Wang is with the Department of Electrical Engineering, National Taiwan University, Taipei 106, Taiwan, R.O.C. (e-mail: wswang@cc.ee.ntu. edu.tw).

Digital Object Identifier 10.1109/JLT.2007.909863

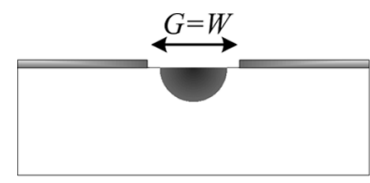

(a)

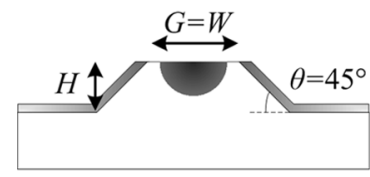

(c)

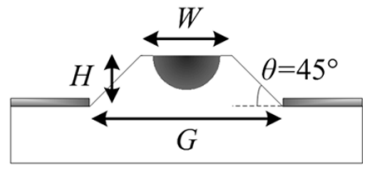

(b)

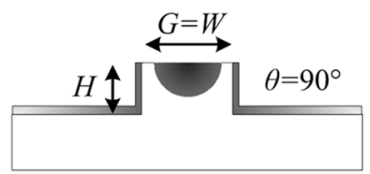

(d)
Fig. 1. Configuration of the EO modulator. (a) Conventional. (b) Chang's [3]. (c) Proposed. (d) Ideal.

where $G$ is the gap of the electrodes, $\lambda$ is the wavelength, $L$ is the length of the electrodes, $r_{33}$ is the EO coefficient, $n$ is the refractive index of the substrate, and $\Gamma$ is the overlap integral, which is given by

$$
\Gamma=\frac{G}{V} \frac{\iint E_{e}\left|E_{o}\right|^{2} d A}{\iint\left|E_{o}\right|^{2} d A} \leq 1
$$

where $V$ is the applied voltage, and $E_{e}$ and $E_{o}$ are the electric and optical fields, respectively. Fig. 1 shows the schematic of various EO modulators. The conventional EO modulator is shown in Fig. 1(a). In addition, a ridged EO modulator reported by Chang et al. [3] is depicted in Fig. 1(b). In this modulator, a ridge of height $H$, top width $W$, and bottom width $G$ is formed by wet etching. Note that the electrodes are placed on both sides of the ridge with a minimum spacing equal to the bottom width of the ridge. The proposed modulator is sketched in Fig. 1(c), where the electrodes are extended to the sidewalls of the ridge such that the minimum spacing between the electrodes is the top width of the ridge. Fig. 1(d) shows an ideal case of the proposed EO modulator, where the angle of the ridge slope $\theta$ is $90^{\circ}$. Practically, the ideal ridge structure can be fabricated by dry etching such as focused ion beam etching. For comparison, practical values of the parameters such as $L=2 \mathrm{~cm}, W=11 \mu \mathrm{m}$, and $\theta=45^{\circ}$ are chosen.

For a rigorous analysis of the proposed EO modulator as shown in Fig. 1(c) and (d), the calculation of the overlap integral consists of numerical simulations of electric and optical fields. Conventionally, the electric field is obtained by solving the Poisson equation with the aid of conformal mapping [5], and the optical field is obtained by assuming a Gaussian index profile [6]. However, there are two problems for the conventional 


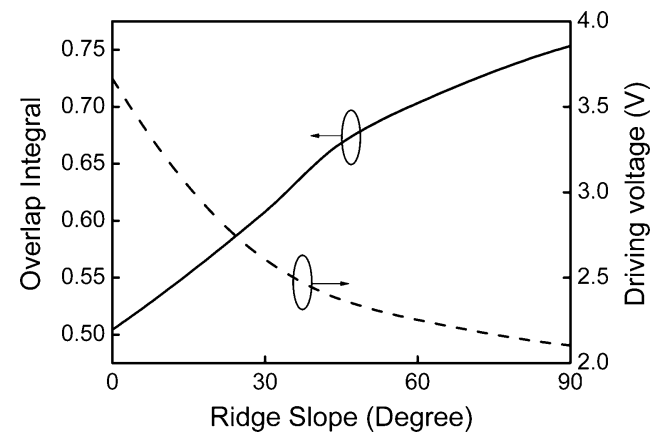

Fig. 2. Overlap integral of the proposed modulator versus angle of the ridge slope for $H=3.5 \mu \mathrm{m}$.

simulation of the overlap integral. First, many electric-field simulation methods have difficulties on dealing with the fringing fields [6] and scaling the simulation window [5]. Second, analytic solution of impurity diffusion distribution is too complicated to be useful for the proposed EO modulator. Moreover, for an analytic solution of the impurity distribution, the unlimited boundaries are usually assumed. However, for a small electrode gap like those shown in Fig. 1(c) and (d), the effects of boundaries cannot be neglected. Therefore, a more general simulation scheme for the overlap integral is needed.

First, the Helmholtz equation is solved with a refractive index distribution that is obtained by solving the diffusion equation as given by

$$
\frac{\partial N}{\partial t}=\vec{\nabla} \cdot(D \vec{\nabla} N)
$$

where $N$ is the impurity (e.g., Ti) concentration, and $D$ is the diffusion coefficient. The impurity concentration is then converted to a refractive index distribution with known relations [7].

The static field is obtained by solving the Poisson equation as follows:

$$
\vec{\nabla} \cdot(\varepsilon \vec{E})=-\frac{\rho}{\varepsilon_{0}}=0
$$

where $\varepsilon$ is the dielectric constant, and $\rho$ is the electric charge density. With the solutions of electric and optical fields, the overlap integral can be calculated to obtain the half-wave voltage. In this paper, $\mathrm{C}++$ and Matlab were used to implement the programs.

Due to lateral chemical etching, the ridge angle is not straight. Fig. 2 shows the angular dependence of the overlap integral for $H=3.5 \mu \mathrm{m}$ and $G=11 \mu \mathrm{m}$. As can be seen from the figure, a larger angle $\left(\theta<90^{\circ}\right)$ gives rise to a larger overlap integral. That is because the horizontal field components are increased with the slope angle. Fig. 3 shows the dependence of the overlap integral and half-wave voltage on the ridge height for various ridge angles. The results show that the overlap integral of modulators in Fig. 1(c) and (d) is gradually increased with the ridge height. However, too high a ridge does not significantly improve the overlap integral. That is because the interaction between the optical and static electric fields is saturated with an increasing ridge height.

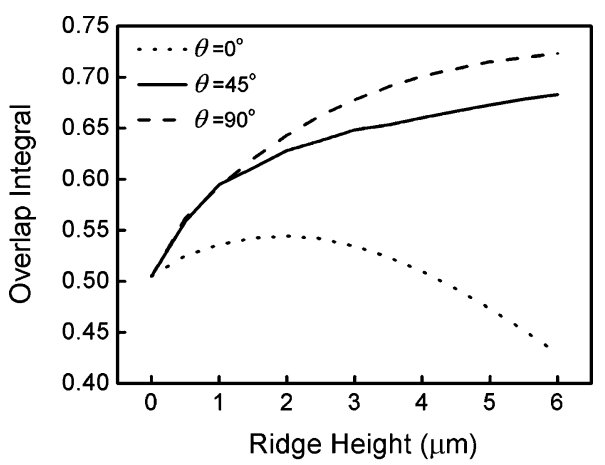

(a)

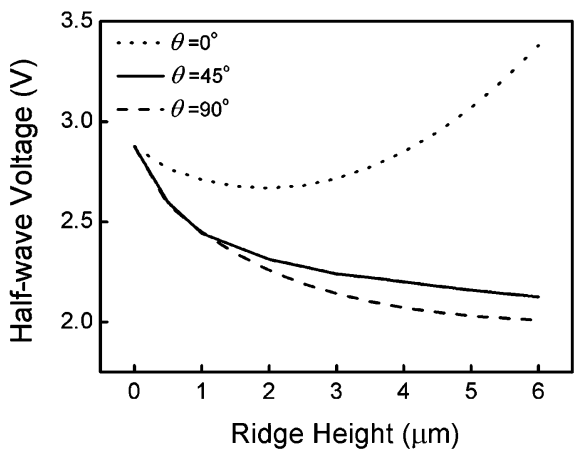

(b)

Fig. 3. (a) Overlap integral and (b) half-wave voltage versus ridge height for $\theta=0^{\circ}, 45^{\circ}$, and $90^{\circ}$.

For comparison, the half-wave voltages of various electrode configurations of modulators are calculated, as shown in Table I. In particular, the proposed and Chang's electrode configurations [3] are extensively compared. The driving voltages as functions of the electrode gap are shown in Fig. 4. It is obvious that the driving voltage of the proposed electrode configuration is improved by $14.6 \%-15.1 \%$. Fig. 5 shows the dependence of the overlap integral and half-wave voltage on the ridge height. As can be seen, for a $1-\mu \mathrm{m}$-high ridge, the overlap integral and the half-wave voltage for the proposed electrode configuration are 0.59 and $2.45 \mathrm{~V}$, respectively. Those for Chang's electrode configuration are 0.51 and $2.85 \mathrm{~V}$, respectively. The improvement of the overlap integral and the half-wave voltage are $15.7 \%$ and $16.3 \%$, respectively. Note that, for Chang's electrode configuration, when the ridge is too high, the overlap integral and half-wave voltage become less improved. That is because the interaction between the optical and static electric fields is decreased with an increasing ridge height. However, for the proposed electrode configuration, this disadvantage is not seen.

Moreover, with the proposed electrode configuration, the area of a Mach-Zehnder modulator with a branching angle of $1^{\circ}$ driven by a voltage of $2.5 \mathrm{~V}$ is $0.23 \mathrm{~mm}^{2}$, but that of the conventional device shown in Fig. 1(a) is $0.28 \mathrm{~mm}^{2}$, which is improved by about $18 \%$. Thus, the chip size can be significantly reduced.

\section{EXPERIMENTAL VERIFICATION}

A push-pull Mach-Zehnder modulator with the proposed electrode configuration, as shown in Fig. 1(c), on an X-cut lithium niobate substrate operating at $1.55 \mu \mathrm{m}$ is chosen for verification. 
TABLE I

Performance of VArious Electro-Optical Modulators

\begin{tabular}{c|cc|c|c}
\hline Data Type & \multicolumn{3}{|c|}{ Simulation } & \multicolumn{2}{|c}{ Experiment } \\
\hline $\begin{array}{c}\text { Electrode } \\
\text { Configuration }\end{array}$ & 11 & 11 & 11 & 11 \\
\hline$W(\mu \mathrm{m})$ & 11 & 14 & 11 & 11 \\
$G(\mu \mathrm{m})$ & $\mathrm{NA}$ & 45 & 45 & 45 \\
$\theta\left({ }^{\circ}\right)$ & 20 & 20 & 20 & 20 \\
$L(\mathrm{~mm})$ & $\mathrm{NA}$ & 1.3 & 1.3 & 1.3 \\
$H(\mu \mathrm{m})$ & 0.501 & 0.505 & 0.611 & 0.607 \\
\hline$\Gamma$ & 2.88 & 2.87 & 2.40 & 2.50 \\
\hline$V_{\pi}(\mathrm{V})$ & & & &
\end{tabular}

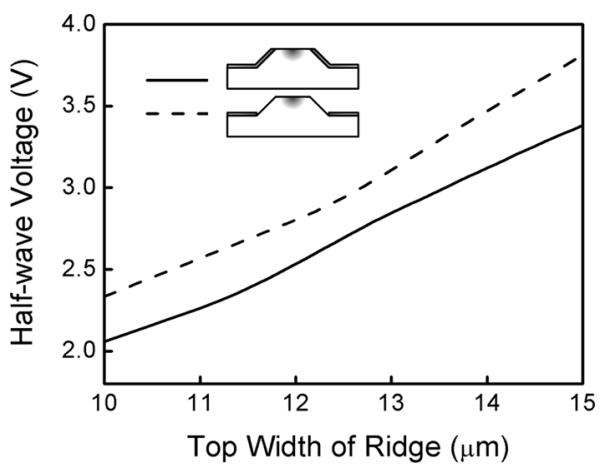

Fig. 4. Half-wave voltage versus electrode gap for $H=2 \mu \mathrm{m}$.

The fabrication process of the Mach-Zehnder modulator is illustrated in Fig. 6. Notice that only one arm of the Mach-Zehnder modulator is shown for simplicity. First, a tantalum strip with a thickness of $100 \mathrm{~nm}$ is coated on a lithium niobate substrate. Then, the proton exchange process is performed by heating the substrate to $200{ }^{\circ} \mathrm{C}$ in a $0.1 \%$ lithium benzoate with benzoic acid as the solvent [8]. The substrate is etched with a mixture of hydrofluoric and nitric acids (1:2) to form a ridge with a height of $1.3 \mu \mathrm{m}$. In steps (d) and (e), the titanium film is patterned to form a strip with a width of $7 \mu \mathrm{m}$ and then diffused at $1020^{\circ} \mathrm{C}$ for $280 \mathrm{~min}$ to form the waveguides. In the last two steps (f) and (g), an oxide layer with a thickness of $100 \mathrm{~nm}$ is deposited as the buffer layer to prevent the optical power loss due to the metallic electrodes. Finally, an aluminum layer with a thickness of $200 \mathrm{~nm}$ is deposited by thermal evaporation and then patterned to form a pair of electrodes with a gap of $11 \mu \mathrm{m}$ and a length of $2 \mathrm{~cm}$ for the Mach-Zehnder modulator.

To check if the electrodes are placed at the position as required, Fig. 7 shows a scanning electron microscopy image of the electrodes. The dark region indicates the lithium niobate, namely the ridge structure, and the light region indicates the electrodes. It is obvious that the electrodes have been correctly

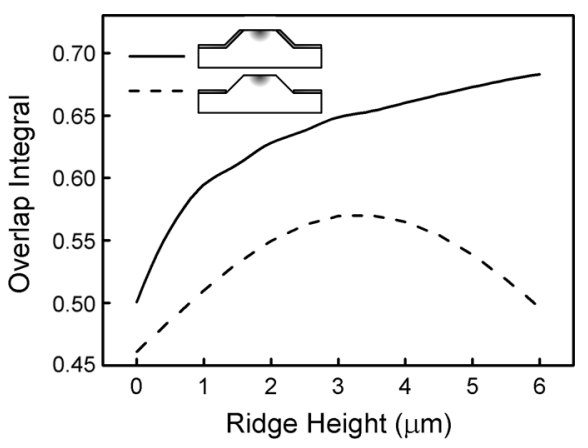

(a)

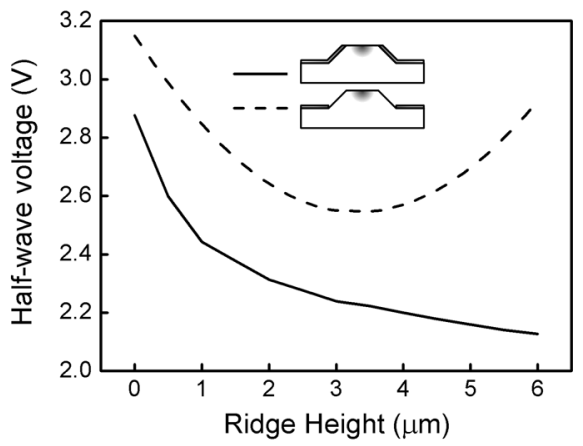

(b)

Fig. 5. (a) Overlap integral and (b) half-wave voltage versus ridge height of modulators in Fig. 1(b) and (c).

aligned and placed. Fig. 8 shows the output optical intensity distribution, which is quite similar to the simulated one. The optical losses of the ridge waveguides are 5-7 dB depending on the ridge height [9].

The measurement system consists of the following components. First, a He-Ne laser with a wavelength of $0.6328 \mu \mathrm{m}$ is used for optical alignment. Then, an infrared laser with a wavelength of $1.55 \mu \mathrm{m}$ is focused at the input end of the waveguide. The input laser beam is transmitted through the waveguide and split to the charge-coupled device (CCD) camera and 


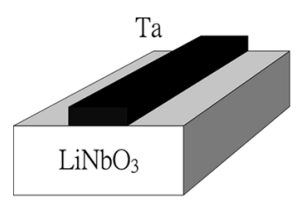

(a)

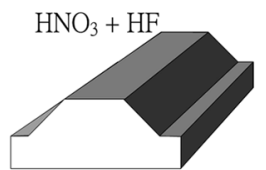

(c)

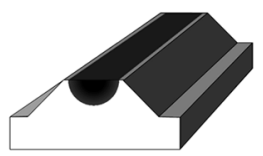

(e)

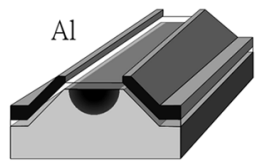

(g) (b)

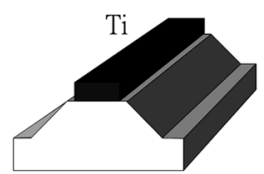

(d)

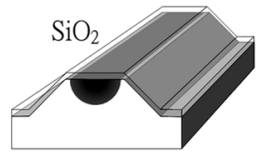

(f)

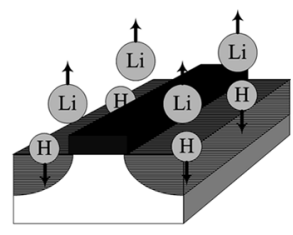

Fig 6. Fabrication process of a ridged EO modulator. (a) Tantalum mask deposition. (b) Proton exchange. (c) Wet etching. (d) Titanium stripe deposition. (e) Diffusion. (f) Silicon oxide buffer layer deposition. (g) Aluminum electrode deposition.

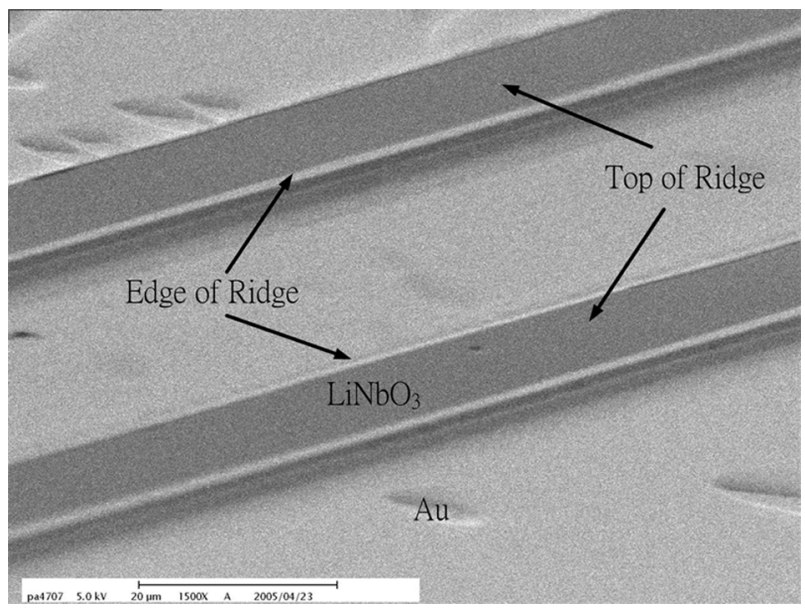

Fig. 7. Top view of ridged waveguides and electrodes.

the detector at the output end. Applying a sawtooth wave on the electrodes, we can see that the output optical power is varying with time on both the CCD camera and the detector. Fig. 9 depicts the optical response of the push-pull Mach-Zehnder modulator driven by a triangular wave. The overlap integral and the half-wave voltage obtained from the experimental data are also shown in Table 1. In comparison to the simulation results, the errors in the overlap integral and the half-wave voltage are $0.6 \%$ and $4.0 \%$, respectively. This shows that the experimental and simulation results are in good agreement. Thus, with the proposed electrode configuration, an improved EO modulator can be realized.

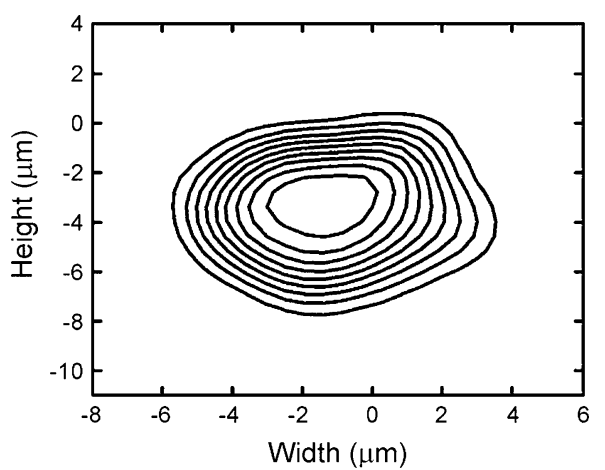

Fig. 8. Measured field intensity distribution of a ridge waveguide.

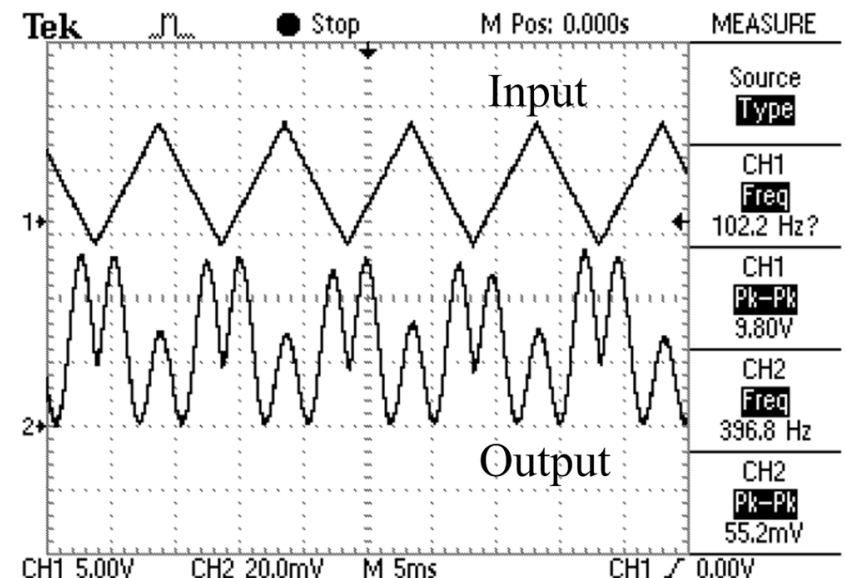

Fig. 9. Optical response of the proposed push-pull Mach-Zehnder modulator driven by a sawtooth wave.

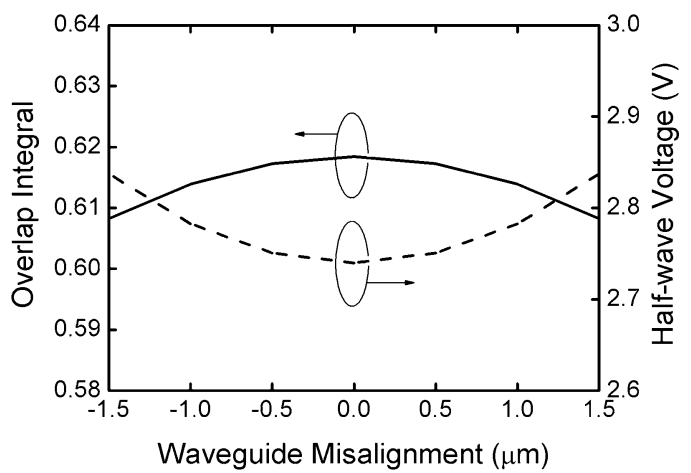

Fig. 10. Overlap integral versus misalignment for $H=2 \mu \mathrm{m}$ and $W=$ $13 \mu \mathrm{m}$.

\section{DISCUSSION}

It is of interest to know the effect of waveguide and electrode misalignment. It has been shown that the overlap integral can be increased when the waveguide is placed a certain offset to the central position of the electrodes [1], [5]. For a ridge with a height of $2 \mu \mathrm{m}$ and a top width of $13 \mu \mathrm{m}$, the overlap integral with various offsets is sketched in Fig. 10. As can be seen, the variation of the overlap integral caused by an offset of $1.5 \mu \mathrm{m}$ for Chang's and the proposed structures are $1.3 \%$ and $1.8 \%$, respectively. These results show, though it was reported that the strength of the electric field is strong near the electrode edges, that the effect of offset is negligible for the improvement of the half-wave voltage. That is because the electrodes of the proposed device have been extended to the sidewall of the ridge, 
so the electric lines are more uniformly distributed. Therefore, the overlap integral can hardly be influenced by any small offset of the waveguide location.

\section{CONCLUSION}

In conclusion, for the enhancement of the performance of EO modulators, a simple modification of the previous electrode configuration has been proposed. With the proposed electrode configuration, smaller device, lower driving voltage, and reasonable wide bandwidth can be achieved. To simulate the proposed electrode configuration, a rigorous analysis has been utilized to calculate the driving voltage. For a ridge with a height of $1.3 \mu \mathrm{m}$, experimental results show that the driving voltage is reduced by $15 \%$ as compared with that of the EO modulator without extended electrode configuration. Details of the application of the proposed EO modulator will be of interest for future study.

\section{REFERENCES}

[1] R. A. Becker and B. E. Kincaid, "Improved electrooptic efficiency in guided-wave modulators," J. Lightw. Technol., vol. 11, no. 12, pp. 2076-2079, Dec. 1993.

[2] R.-S. Cheng, W.-L. Chen, and W.-S. Wang, "Mach-Zehnder modulators with lithium niobate ridge waveguides fabricated by proton-exchange wet etch and nickel indiffusion," IEEE Photon. Technol. Lett., vol. 7, no. 11, pp. 1282-1284, Nov. 1995.

[3] S.-J. Chang, C.-L. Tsai, Y.-B. Lin, J.-F. Liu, and W.-S. Wang, "Improved electrooptic modulator with ridge structure in $\mathrm{X}$-cut $\mathrm{LiNbO}_{3}$," J. Lightw. Technol., vol. 17, no. 5, pp. 843-847, May 1999.

[4] R. C. Alferness, "Waveguide electrooptic modulators," IEEE Trans. Microw. Theory Tech., vol. MTT-30, no. 8, pp. 1121-1137, Aug. 1982.

[5] D. Marcuse, "Electrostatic field of coplanar lines computed with the point matching method," IEEE J. Quantum Electron., vol. 25, no. 5, pp. 939-947, May 1989.
[6] C. M. Kim and R. V. Ramaswamy, "Overlap integral factors in integrated optic modulators and switches," J. Lightw. Technol., vol. 7, no. 7, pp. 1063-1070, Jul. 1989.

[7] S. Fouchet, A. Carenco, C. Daguet, R. Guglielmi, and L. Riviere, "Wavelength dispersion of Ti induced refractive index change in $\mathrm{LiNbO}_{3}$ as a function of diffusion parameters," J. Lightw. Technol., vol. LT-5, no. 5, pp. 700-708, May 1987.

[8] F. Laurell, J. Webjörn, G. Arvidsson, and J. Holmberg, "Wet etching of proton-exchanged lithium niobate-A novel processing technique," J. Lightw. Technol., vol. 10, no. 11, pp. 1606-1609, Nov. 1992.

[9] T.-L. Ting, L.-Y. Chen, and W.-S. Wang, "A novel wet-etching method using joint proton source in $\mathrm{LiNbO}_{3}$," IEEE Photon. Technol. Lett., vol. 18, no. 4, pp. 568-570, Feb. 2006.

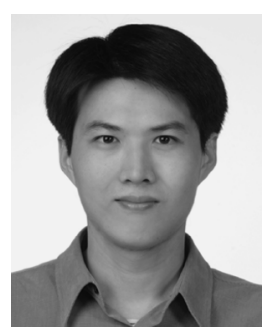

Yi-Kuei Wu (S'04) was born in Taipei, Taiwan, R.O.C., on November 3, 1980. He received the B.S. degree in electrical engineering and the M.S. degree in electrooptical engineering from the $\mathrm{Na}$ tional Taiwan University, Taipei, in 2003 and 2005, respectively.

$\mathrm{He}$ is currently with the Graduate Institute of Electro-Optical Engineering, National Taiwan University.

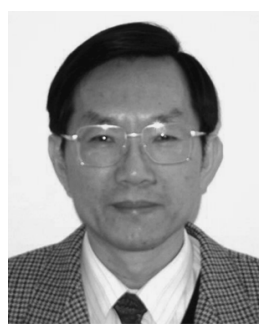

Way-Seen Wang (M'84) was born in Taipei, Taiwan, R.O.C., on March 11, 1948. He received the B.S. degree in electrical engineering from the National Taiwan University (NTU), Taipei, in 1970, and the M.S. and Ph.D. degrees from the University of Southern California, Los Angeles, in 1975 and 1979, respectively.

Since August 1971, he has been with the Department of Electrical Engineering, NTU, where he became a Full Professor in 1984. His current research interest includes the design and fabrication of integrated optical waveguide devices. 